\title{
Autoeficacia y adherencia terapéutica en personas con diabetes mellitus tipo 2
}

Self-efficacy and adherence to treatment in people with type 2 diabetes mellitus

\author{
Joel Omar González-Cantero", Ma. de los Ángeles González Á., José Vázquez C., Sergio, Galán C. \\ Universidad de Guadalajara, Jalisco, México
}

(RECIBIDO 10/03/2015, AcEPTADo 15/06/2015)

\begin{abstract}
RESUMEN
La diabetes mellitus 2 (DM2) se agudiza cuando las personas no se adhieren a su tratamiento. Se requiere evaluar los factores que influyen favorablemente al logro de la adherencia terapéutica. Objetivo. Evaluar la relación entre la autoeficacia y la adherencia terapéutica en pacientes con DM2 atendidos en la Unidad de Medicina Familiar No. 48 (UMF 48) del Instituto Mexicano del Seguro Social (IMSS). Método. Descriptivo - correlacional con 314 participantes. Se aplicaron la Escala de Adherencia al Tratamiento de la Diabetes Mellitus tipo II versión III (EATDM - III) y el Spanish Diabetes Self-Efficacy. Resultados. La autoeficacia se relacionó positiva y significativamente con la adherencia terapéutica $(\mathrm{r}=.400$, $\mathrm{p}<0.01$ ). Las personas autoeficaces fueron 3.46 veces más adherentes a su tratamiento que las no autoeficaces $(\mathrm{RM}=3.46, \mathrm{p}<.05)$. Discusión y conclusiones. Los hallazgos de esta investigación indican que la autoeficacia tiene un efecto favorable para la adherencia terapéutica en esta población de estudio. Se sugiere realizar intervenciones para fortalecer la autoeficacia de las personas respecto al tratamiento de la DM2.
\end{abstract}

Palabras clave: Autoeficacia, adherencia terapéutica, diabetes mellitus 2, psicología de la salud.

\begin{abstract}
Diabetes mellitus 2 (DM2) is exacerbated when people do not adhere to their treatment. It requires evaluating the factors that influence favorably the achievement of adherence to treatment. Objective. Evaluate the relationship between self-efficacy and adherence to treatment in patients with DM2 served in Family Medicine Unit No. 48 (UMF 48) of the Instituto Mexicano del Seguro Social (IMSS). Method. Descriptive-correlational with 314 participants. Were applied the Scale of Adherence to the Treatment of Diabetes Mellitus Type II version III (EATDM - III) and the Spanish Diabetes Self-Efficacy. Results. Self-efficacy is positively and significantly associated with adherence to treatment $(r=.400, p<0.01)$.
\end{abstract}

1 Profesor de la Universidad de Guadalajara. Colaborador del Centro de Investigación en Comportamiento y Salud de CUValles. E-mail: joelomar@profesores.valles.udg.mx 
People self-efficient were 3.46 times more adherent to their treatment than those who were not self-efficient $(R M=3.46, p<.05)$. Discussion and conclusions. The findings of this research suggest that self-efficacy has a favorable impact on adherence to treatment in this study population. It is suggested to perform interventions to strengthen self-efficacy of individuals with respect to the treatment of the DM2.

Keywords: Self-efficacy, adherence to treatment, diabetes mellitus 2, Health Psychology.

\section{INTRODUCCIÓN}

Actualmente hay en el mundo 346 millones de personas con diabetes mellitus (DM); 33 de ellos en América Latina (OMS, 2011). Particularmente en México la prevalencia de diabetes mellitus tipo 2 (DM2) es de $14.42 \%$ (Villalpando y col., 2010).

El problema de una enfermedad crónica como la DM2 se agudiza cuando las personas no se adhieren a su tratamiento, lo cual provoca, entre sus principales consecuencias, la disminución de la calidad de vida de la persona, aumento del gasto en los servicios sanitarios y aumento de la mortalidad.

Según estima la OMS (2003), el 50\% de las personas con enfermedad crónica (cardiovasculares, diabetes y cáncer) no son adherentes a su tratamiento en los países desarrollados, situación que se agrava en los subdesarrollados por la falta de acceso a los medicamentos.

En México, el déficit en la adherencia al tratamiento de la DM coloca a esta enfermedad como el principal motivo de atención en el Instituto Mexicano del Seguro Social (IMSS), que provee atención sanitaria a cerca del $60 \%$ de la población mexicana.

El impacto económico que provoca la atención de la DM y otras enfermedades crónico-degenerativas puede colapsar en un futuro el sistema sanitario mexicano. Al respecto, hay que considerar que en los últimos 40 años se pasó de ser un país de gente joven a ser un país con mayor proporción de adultos y personas de la tercera edad. Esto repercutiría en una mayor prevalencia de la DM2 y otras enfermedades crónico-degenerativas que no conducen a la muerte inmediata, pero que son grandes consumidoras de servicios de salud, y, en ese sentido, se tendría un impacto financiero alto (Vázquez y Panduro, 2001).

Muestra de ello es que en el IMSS, durante los noventa, la DM ocupó el primer lugar como egreso hospitalario y reportó que la tasa de complicaciones asociadas a la enfermedad, en particular renales y de la circulación, fue en aumento (Escobedo y Verdín, 1996).

La adherencia terapéutica tiene muchas aristas y, como señala Martín (2004), es una "conducta compleja que consta de una combinación de aspectos propiamente conductuales, unidos a otros relacionales y volitivos que conducen 
a la participación y comprensión del tratamiento por parte del paciente y del plan para su cumplimiento, de manera conjunta con el profesional de la salud, y la consiguiente respuesta modulada por una búsqueda activa y consciente de recursos para lograr el resultado esperado".

En consonancia con lo anterior, la adherencia a la terapéutica implica para las personas con DM2 restricciones en sus actividades, cambios en la dieta, dejar de fumar y la necesidad de hacer actividad física, lo cual suscita alteraciones en muchos aspectos de sus vidas.

Para lograr elevar los niveles de adherencia terapéutica, se requiere, además de la modificación de comportamientos de la persona, de la participación de los profesionales de la salud y modificaciones en el sistema sanitario en el primer nivel de atención.

En lo psicológico, el problema de la adherencia terapéutica está estrechamente relacionado con aspectos subjetivos y comportamentales que deben considerarse en el curso y tratamiento.

La simple transmisión de información respecto al tratamiento que debe llevar una persona con diabetes no es suficiente para un afrontamiento adecuado de la enfermedad y su tratamiento. Algunos trabajos (Hurley y Shea, 1992; Glasgow y Osteen, 1992) ya han puesto de manifiesto que resulta insuficiente el conocimiento del tratamiento, pues, aunque necesario, por sí solo no es predictor del comportamiento de autocuidado deseado.

Partiendo de lo anterior, se ha indagado sobre los factores que pueden favorecer la adherencia terapéutica. Dentro de los principales, se le ha asociado con el estrés (Garay-Sevilla y col., 2000) y el apoyo social (Arredondo, Márquez, Moreno y Bazán, 2006). Los resultados han mostrado, en el primer caso, que el estrés no está directamente involucrado con la adherencia terapéutica, sino que se relaciona con la obesidad y el control metabólico; y en el segundo, respecto al apoyo social, se ha encontrado una correlación negativa entre el apoyo social y los niveles de glucemia; por ello, como lo explica Ginarte (2001), el apoyo social bien puede contribuir a que la adherencia terapéutica incremente o disminuya.

Una variable que ha resultado más consistente para favorecer la adherencia terapéutica es la autoeficacia. Este concepto emana de la teoría sociocognitiva. Bandura (2006) señala que está determinada por diversos factores cognitivos y sociales, cuya presencia puede variar según las características socioculturales de una población determinada y la define como "las creencias en las propias capacidades para organizar y ejecutar los cursos de acción requeridos para manejar situaciones futuras".

Acorde a la teoría de la autoeficacia, las expectativas de eficacia y de resultados modulan el efecto terapéutico de algunos tratamientos psicológicos y constituyen algunas conductas relacionadas con la salud. 
En estudios realizados en personas con DM, se ha encontrado que las creencias de autoeficacia tienen una gran relevancia para alcanzar las primeras metas del tratamiento (Aljasem, Peyrot, Wissow y Rubin, 2001; Rose, Fliege, Hildebrandt, Schirop y Klapp, 2002); además de fungir como predictoras de la adherencia a regímenes prescritos en adultos con DM2, después de controlar las creencias de salud y las percepciones de las barreras (Ferrer, Gili y Durán, 1999; Lorig, Ritter y Jacquez, 2005; Senecal, Nouwen y White, 2000).

Por otro lado, partiendo de que para el tratamiento de la DM2 es necesario hacer una dieta saludable, realizar ejercicio o actividad física regular y tener comportamiento de autocuidado, algunos estudios han analizado la autoeficacia y su relación con tales comportamientos.

$\mathrm{Al}$ respecto de la dieta, varios trabajos han señalado la influencia favorable de la autoeficacia (Bartfield, Ojehomon, Huskey, Davis y Wee, 2010; Warziski, Sereika, Styn, Music y Burke, 2008).

En el caso del ejercicio o actividad física, los resultados son similares pues se han encontrado relaciones directas entre la autoeficacia y la adherencia a lo prescrito (Annesi, Unruh, Marti, Gorjala y Tennant, 2011; Jones, Harris, Waller y Coggins, 2005; Plotnikoff, Brez, y Hotz, 2000).

Por otra parte, también se coincide en la relación favorable de la autoeficacia con el comportamiento de autocuidado (Balcázar, Gurrola, Urcid y Colín, 2011; Wu y col., 2007).

Por ello, ante la ausencia de investigaciones en la región en las cuales se identifiquen los niveles de la autoeficacia y la adherencia terapéutica y su posible relación, en personas con DM2 de la región Occidente de México, el presente trabajo tuvo como objetivo evaluar la relación entre la autoeficacia y la adherencia terapéutica en pacientes con DM2 atendidos en la Unidad de Medicina Familiar No. 48 (UMF 48) del IMSS. Se considera que aquellas personas con DM2 que tengan mayores niveles de autoeficacia tendrán también mayor adherencia terapéutica.

\section{MÉTODO}

\section{Diseño}

Se realizó un estudio de tipo transversal con un alcance descriptivo-correlacional. Transversal, porque la recolección de datos se realizó solo una vez. Descriptivocorrelacional, porque se pretende medir variables, explicar y cuantificar sus relaciones en aras de esbozar algunas predicciones y dar bases a estudios empíricos y explicativos. 


\section{Selección y tamaño de la muestra}

Se eligió la UMF 48 del IMSS debido a que es la más grande en la región Occidente de México. Se eligió un muestreo probabilístico sistemático. Se calculó con el programa informático Decision Analyst STATS 2.0, dando como resultado 371 participantes. La tasa de no respuesta fue del $15.36 \%$ (57 sujetos), quedando así una muestra final de 314 participantes. En el cálculo de la muestra se consideró un nivel de confianza del $95 \%$ y una precisión del $5 \%$.

\section{Participantes}

Los criterios de inclusión de los participantes fueron padecer DM2; tener más de 19 años de edad; tener 1 o más años con el diagnóstico y aceptar participar en el estudio firmando el consentimiento informado. La muestra se conformó por $206(65.6 \%)$ mujeres y $108(34.4 \%)$ hombres. La edad tuvo un rango de entre 23 y 90 años con una $\bar{x}=61.66$ años, $D E=10.64$. Respecto al estado civil, 91 participantes eran solteros $(29 \%)$ y $223(71 \%)$ casados. En torno al nivel educativo, el $73.6 \%$ de los participantes tiene educación primaria, el $15 \%$ educación secundaria, el $6.7 \%$ preparatoria y solo el $4.8 \%$ licenciatura. En relación con el tiempo de padecimiento de la enfermedad, se halló en la muestra un mínimo de 1 año y un máximo de 40 años con la enfermedad, con una $\overline{\mathrm{X}}=$ 10.46, $\mathrm{DE}=8.21$. Respecto al IMC, $36.6 \%(\mathrm{~N}=115)$ tenía sobrepeso; $27.7 \%$ obesidad grado I $(\mathrm{N}=87) ; 8.9 \%$ obesidad grado II $(\mathrm{N}=28)$ y $2.5 \%$ obesidad grado III $(\mathrm{N}=8)$. En suma, el $75.7 \%$ tenía sobrepeso u obesidad. El 23.2\% (N $=73)$ tenía normopeso y el $1 \%$ infrapeso $(\mathrm{N}=3)$.

\section{Instrumentos}

La Escala de Adherencia al Tratamiento de la Diabetes Mellitus tipo II versión III (EATDM - III) (Villalobos y Araya, 2001). Es una escala tipo Likert de 55 reactivos que se conforma por 7 factores comportamentales: 1) apoyo familiar; 2) organización y apoyo comunal; 3) ejercicio físico; 4) control médico; 5) higiene y autocuidado; 6) dieta y 7) valoración de la condición física. Las opciones de respuesta son: 0: "Nunca lo hago"; 1: "Casi nunca lo hago" (entre el 1\% y el $33 \%$ de las ocasiones); 2: "Lo hago regularmente" (entre el 34\% y el 66\% de las ocasiones); 3: "Casi siempre lo hago" (entre el 67\% y el 99\% de las ocasiones); 4: "Siempre lo hago" (el 100\% de las ocasiones). El índice de confiabilidad Alfa de Cronbach ha ido de .87 (Villalobos y Araya, 2001) a .88 (Villalobos y col., 2006; Quirós y Villalobos, 2007).

Spanish Diabetes Self-Efficacy (Stanford Patient Education Research Center, 2004). Se diseñó específicamente para evaluar la autoeficacia de las personas 
con DM2. Es una escala de tipo continua del "1 al 10", donde "1" es "Muy inseguro(a)" y "10" es "Muy seguro(a)", compuesta por 8 reactivos. La puntuación de la escala es la media de los ocho ítems. El índice de confiabilidad Alfa de Cronbach reportado por los creadores de la escala fue de .85 , obtenido de una muestra con 189 personas con DM2.

\section{Procedimiento}

El proyecto se presentó en la Coordinación Clínica de Educación e Investigación en Salud de la UMF 48 del IMSS. Con la autorización debida, se pidió la colaboración de los asistentes médicos de cada consultorio para identificar a las personas que cubrían los requisitos para la participación en el estudio mediante la revisión de los expedientes. Primeramente se explicaba a los participantes el objetivo del estudio y el carácter anónimo de sus respuestas en los instrumentos haciendo énfasis en que la investigación se encontraba bajo los lineamientos éticos que señalan el IMSS y la Ley General de Salud respecto a las actividades de investigación. Una vez que aceptaba participar en el estudio y firmaba la carta de consentimiento informado, se aplicaba la entrevista y los instrumentos en las salas de espera de cada consultorio. Se entrenó a 3 estudiantes de Psicología para que colaboraran como aplicadores de los instrumentos. En una primera etapa se realizó un pilotaje con 65 pacientes. Una vez obtenidos los resultados del pilotaje y habiendo tomado en cuenta las observaciones derivadas del mismo, se procedió con la recolección de datos de la muestra requerida.

\section{Análisis de datos}

Los distintos análisis estadísticos de los datos se realizaron con el paquete estadístico IBM SPSS Statistics 19, el programa Decision Analyst STATS 2.0 y el Epi Info 7. El procedimiento se dividió en tres fases: $1^{\text {a }}$ fase: se obtuvieron los datos descriptivos (frecuencias y porcentajes, medias y desviaciones estándar) de las variables sociodemográficas y de los resultados de los instrumentos utilizados; $2^{\mathrm{a}}$ fase: se realizaron diversas pruebas estadísticas para evaluar la relación de la adherencia terapéutica y la autoeficacia, como el coeficiente de correlación de Pearson $(r)$ y la razón de momios $(R M)$ y $3^{\text {a }}$ fase: se calculó el Alpha de Cronbach para establecer la confiabilidad tanto de la Escala de Adherencia al Tratamiento de la Diabetes Mellitus tipo II versión III como del Spanish Diabetes Self-Efficacy en la muestra de este estudio.

\section{RESULTADOS}

En torno a la autoeficacia, el $81.5 \%(\mathrm{~N}=256)$ de los participantes son "autoeficaces", mientras el $18.5 \%(\mathrm{~N}=58)$ resultaron "no autoeficaces". Respecto a la adherencia al tratamiento, el $78.3 \%(\mathrm{~N}=246)$ de las personas se ubicaron como "no adherentes" y el $21.7 \%(\mathrm{~N}=68)$ como "adherentes" a su tratamiento. 
Figura 1. Distribución de la muestra por autoeficacia y adherencia terapéutica

No autoeficaces

$18.5 \%$
Autoeficaces

$81.5 \%$
No adherentes

$78.3 \%$
Adherentes

$21.7 \%$

Los participantes obtuvieron respecto a la autoeficacia una $\overline{\mathrm{x}}=7.38, \mathrm{DE}=$ 1.87; lo cual corresponde a un nivel medio, dentro del rango que se considera autoeficaz. El reactivo 7, que se refiere a la confianza que la persona tiene para evaluar cuando algún cambio en su enfermedad le indica que debe acudir al médico, fue el que obtuvo la media más alta $(\overline{\mathrm{x}}=8.36, \mathrm{DE}=2.54)$, se ubica en un nivel alto de autoeficacia. Por otra parte, el reactivo 1, que versa sobre la confianza que tiene la persona para comer cada 4 o 5 horas al día, tuvo la media más baja de todos los reactivos $(\overline{\mathrm{x}}=6.29$, $\mathrm{DE}=3.39)$; pero, aun así, corresponde a un nivel medio, dentro del rango considerado como autoeficaz.

Tabla 1. Medias y desviaciones estándar de la escala Spanish Diabetes Self-Efficacy

\begin{tabular}{lcc}
\hline \multicolumn{1}{c}{ Reactivo } & $\overline{\mathrm{X}}$ & $\mathrm{DE}$ \\
\hline 1. Comer alimentos cada 4 o 5 horas (diariamente) & 6.29 & 3.39 \\
2. Continuar la dieta preparando o compartiendo alimentos & 7.30 & 3.03 \\
3. Elegir los alimentos adecuados cuando se está hambriento & 7.29 & 2.93 \\
4. Hacer ejercicio de 15 a 30 minutos (4 o 5 veces por semana) & 7.39 & 3.24 \\
5. Prevenir que el nivel de azúcar baje cuando se hace ejercicio & 6.78 & 3.48 \\
6. Saber que hacer cuando el nivel de azúcar sube o baja & 7.49 & 3.12 \\
7. Evaluar los cambios de la enfermedad & 8.36 & 2.54 \\
8. Controlar la diabetes & 8.19 & 2.48 \\
\hline Puntaje total de autoeficacia percibida & 7.38 & 1.87 \\
\hline
\end{tabular}

Los demás reactivos $(2,3,4,6$ y 8$)$ se ubican con un promedio correspondiente a un nivel medio de autoeficacia y con puntajes dentro del rango considerado como autoeficaz. 
En lo que respecta a la adherencia terapéutica, el promedio del total de la muestra fue de $\overline{\mathrm{x}}=58.62, \mathrm{DE}=10.84$, lo que revela que las personas regularmente hacen lo concerniente a su tratamiento en general; sin embargo, este puntaje, de acuerdo a los criterios del autor de la escala, indica que no hay adherencia terapéutica.

Tabla 2. Medias y desviaciones estándar de la EATDM - III

\begin{tabular}{lcc}
\hline \multicolumn{1}{c}{ Factor comportamental } & $\overline{\mathrm{X}}$ & $\mathrm{DE}$ \\
\hline Apoyo familiar & 53.13 & 25.10 \\
Organización y apoyo comunal & 22.65 & 18.55 \\
Ejercicio físico & 54.45 & 18.95 \\
Control médico & 81.61 & 14.52 \\
Higiene y autocuidado & 81.35 & 15.00 \\
Dieta & 75.75 & 16.43 \\
Valoración de la condición física & 64.29 & 37.41 \\
Puntaje total de adherencia al tratamiento & 58.62 & 10.84 \\
\hline
\end{tabular}

Los factores control médico e higiene y autocuidado fueron los que resultaron con los puntajes más altos con una $\overline{\mathrm{X}}=81.61, \mathrm{DE}=14.52$ y $\overline{\mathrm{x}}=81.35, \mathrm{DE}=$ 15.00 , respectivamente, los cuales corresponden al nivel casi siempre lo hago y consecuentemente indican adherencia terapéutica. Asimismo, el factor dieta tuvo una $\overline{\mathrm{X}}=75.75, \mathrm{DE}=16.43$, la cual corresponde también al nivel casi siempre. La valoración de la condición física se encuentra en el nivel regularmente $(\overline{\mathrm{X}}$ $=64.29, \mathrm{DE}=37.41$ ), lo cual no corresponde a un puntaje que se considere como adherente. De la misma forma, los factores ejercicio y apoyo familiar se encuentran en el nivel regularmente. En tanto que el factor organización y apoyo comunal fue el factor más bajo $(\overline{\mathrm{X}}=22.65, \mathrm{DE}=18.55)$ y corresponde al nivel casi nunca lo hago, que implica no adherencia terapéutica en ese rubro.

Por otro lado, con la medida estadística razón de momios $(R M)$ se identificó que las personas autoeficaces fueron 3.46 veces más adherentes a su tratamiento que las no autoeficaces, lo cual resulta significativo $(R M=3.46, p<.05)$. Los demás resultados pueden apreciarse en la tabla 3.

Tabla 3. Asociación entre autoeficacia y factores de la adherencia terapéutica

\begin{tabular}{cccccccc}
\hline & & \multicolumn{2}{c}{ Autoeficaces } & \multicolumn{2}{c}{ No autoeficaces } & & \\
\cline { 3 - 6 } \multicolumn{1}{c}{ Factor } & Adherente & $\mathrm{N}$ & $\%$ & $\mathrm{~N}$ & $\%$ & RM & IC \\
\hline \multirow{2}{*}{ Apoyo familiar } & Sí & 85 & 33.2 & 11 & 19 & \multirow{2}{*}{2.12} & $1.04-4.30 *$ \\
& No & 171 & 66.8 & 47 & 81 & & \\
\hline Organización y & Sí & 10 & 3.9 & 0 & 0 & - & - \\
apoyo comunal & No & 246 & 96.1 & 58 & 100 & & - \\
\hline
\end{tabular}




\begin{tabular}{lccccccc}
\hline \multirow{2}{*}{ Ejercicio físico } & Sí & 68 & 26.6 & 7 & 12.1 & 2.63 & $1.14-6.08 *$ \\
& No & 188 & 73.4 & 51 & 87.9 & & \\
\hline \multirow{2}{*}{ Control médico } & Sí & 230 & 89.8 & 45 & 77.6 & \multirow{2}{*}{2.55} & $1.22-5.34 *$ \\
& No & 26 & 10.2 & 13 & 22.4 & & \\
\hline Higiene y & Sí & 212 & 82.8 & 36 & 62.1 & \multirow{2}{*}{2.94} & \multirow{2}{*}{$1.58-5.48 * * *$} \\
autocuidado & No & 44 & 17.2 & 22 & 37.9 & & \\
\hline \multirow{2}{*}{ Dieta } & Sí & 195 & 76.2 & 39 & 67.2 & \multirow{2}{*}{1.55} & $.83-2.89$ \\
\hline Valoración de la & No & 61 & 23.8 & 19 & 32.8 & & \\
condición física & Sí & 149 & 58.2 & 20 & 34.5 & \multirow{2}{*}{2.64} & $1.45-4.80 * *$ \\
\hline Adherencia al & No & 107 & 41.8 & 38 & 65.5 & & \\
tratamiento & Sí & 63 & 24.6 & 5 & 8.6 & \multirow{2}{*}{3.46} & \multirow{2}{*}{$1.32-9.03 *$} \\
\hline
\end{tabular}

Intervalo de confianza $95 \%, * p<.05 ; * * p<.01 ; * * p<.001$

En lo que concierne a la correlación, con la prueba $r$ de Pearson se identificó que la autoeficacia se relacionó positiva y significativamente con la adherencia terapéutica $(r=.400, p<0.01)$; esto implica que a mayor autoeficacia, también hay mayor adherencia terapéutica. Sin embargo, como se aprecia en la tabla 4, con los factores comportamentales la autoeficacia tiene una relación significativa estadísticamente, pero con coeficientes de correlación bajos.

Tabla 4. Correlación entre la autoeficacia y los factores comportamentales de la adherencia terapéutica

\begin{tabular}{ccc}
\hline Factor comportamental & $\mathrm{r}$ de Pearson & $\mathrm{p}$ \\
\hline Apoyo familiar & .166 & $<.01$ \\
Organización y apoyo comunal & .264 & $<.01$ \\
Ejercicio físico & .338 & $<.01$ \\
Control médico & 184 & $<.01$ \\
Higiene y autocuidado & .254 & $<.01$ \\
Dieta & .178 & $<.01$ \\
Valoración de la condición física & 183 & $<.01$ \\
Total de la escala & .400 & $<.01$ \\
\hline
\end{tabular}

Respecto a la confiabilidad de los instrumentos, la escala Spanish Diabetes SelfEfficacy obtuvo un $\alpha=.76$, en comparación con la que los autores de la escala reportaron $(\alpha=.85)$. La EATDM - III obtuvo un $\alpha=.80$ en general. En la tabla 5 , se muestran los coeficientes de confiabilidad obtenidos en este estudio y su comparación con lo que otros estudios reportan. 
Tabla 5. Alpha de Cronbach de la EATDM - III

\begin{tabular}{ccccc}
\hline Factor & Estudio & Villalobos* & Villalobos** & Quirós*** \\
\hline Apoyo familiar & .81 & .91 & .84 & .84 \\
Organización y apoyo comunal & .70 & .88 & .83 & .78 \\
Ejercicio físico & .58 & .89 & .83 & .82 \\
Control médico & .39 & .80 & .78 & .78 \\
Higiene y autocuidado & .32 & .64 & .70 & .56 \\
Dieta & .72 & .89 & .90 & .89 \\
Valoración de la condición física & .67 & .93 & .83 & .85 \\
Total de la escala & .80 & .87 & .88 & .88 \\
\hline
\end{tabular}

*Villalobos y Araya (2001); **Villalobos y col. (2006); ***Quirós y Villalobos (2007)

\section{DISCUSIÓN}

Los resultados de estudio coinciden con otros similares realizados en otros lados del mundo (Nyunt y col., 2010; O'Hea y col., 2009; Dit, Baban y Dumitrascu, 2012), en donde se ha reportado que la autoeficacia estaba asociada con el control glucémico. En el primero de ellos, el $62 \%$ de los pacientes con DM2 de esa muestra tenía altos niveles de autoeficacia y solo el $27.1 \%$ tenía un adecuado control glucémico; lo cual concuerda con el estudio que nos ocupa, en donde la asociación entre autoeficacia y adherencia al tratamiento es altamente significativa y la mayoría de la muestra tiene alta autoeficacia $(81.5 \%)$, pero, aun así, un porcentaje bajo (21.7\%) es adherente.

Dit, Baban y Dumitrascu (2012) encontraron en su muestra altos niveles de autoeficacia para tomar medicamentos prescritos, en tanto que en la presente investigación el factor control médico tuvo la media más alta de todos los factores que componen la escala de adherencia. Esto puede explicarse considerando que al igual que en el estudio realizado en Rumania, en el que nos ocupa se realizó en una institución en donde hay un programa nacional gratuito para abastecer de los medicamentos necesarios y ofrecer un programa de citas mensuales para los pacientes. Se esboza entonces, como lo señala Harris (2001), que la falta de control médico puede deberse a características de los sistemas de salud de cada región, pero en estos casos no hay tal déficit y, por ello, sí altos porcentajes de control médico.

No obstante, es insoslayable considerar que un alto control médico no implica que las personas con DM2 tengan una dieta adecuada y realicen el ejercicio físico suficiente como lo marca el tratamiento integral de la diabetes. 
En el presente estudio, la autoeficacia tuvo una relación significativa con la dieta y el ejercicio, lo que coincide con lo reportado por McCleary-Jones (2011), y también resultó una relación significativa con el autocuidado, al igual que en el trabajo de Sarkar, Fisher y Schillinger (2006). De esta forma, se pone de manifiesto que la autoeficacia tiene una asociación positiva con los tres principales componentes del tratamiento en DM2 (dieta, ejercicio físico y autocuidado).

Por otra parte, respecto a la adherencia terapéutica, el ejercicio físico tiene puntajes bajos, pues tan solo cerca del $24 \%$ de la muestra en el presente estudio se adhiere a las conductas comprendidas en ese factor, lo cual es coincidente con lo reportado por Dit, Baban y Dumitrascu (2012); sin embargo, respecto a la dieta, ellos refieren también puntajes bajos de adherencia a ella, en tanto que en la presente investigación el porcentaje de adherencia que obtuvieron los pacientes estuvo de cerca del 75\%. Cabe señalar que el reactivo 1 (comer 4 o 5 veces al día) de la Spanish Diabetes Self-Efficacy tuvo la media más baja, con 6.29, y ligeramente alcanza el puntaje para ser considerado como eficaz, lo cual puede dar cuenta de que los pacientes pueden experimentar alguna complicación con las cantidades y el número de veces adecuados de ingestión, y no tanto con el tipo de alimentos.

En torno al apoyo y organización comunal y el apoyo familiar, ambos presentaron puntajes muy bajos, lo cual puede explicarse por factores políticos, sociales y culturales; por ejemplo, las políticas públicas en salud, las prácticas comunitarias y las creencias.

En relación con los instrumentos, la escala Spanish Diabetes Self-Efficacy se puede considerar un instrumento confiable, pues está diseñada específicamente para DM2 y obtuvo un Alpha de Cronbach aceptable. Por otra parte, la Escala de Adherencia al Tratamiento de la Diabetes Mellitus tipo II versión III se puede considerar confiable en lo general. Sin embargo, algunos factores no tuvieron coeficientes de confiabilidad aceptables (control médico, higiene y autocuidado y ejercicio físico).

\section{CONCLUSIONES}

Los hallazgos de este estudio respecto a la autoeficacia y la adherencia terapéutica sugieren que es bastante útil fortalecer la autoeficacia de las personas para lograr que se adhieran a su tratamiento; por ende, resulta útil incluir aspectos de la teoría de la autoeficacia en el diseño de programas psicoeducativos para el paciente con DM2.

Pese a que se demuestra la relación que guarda la autoeficacia y la adherencia terapéutica, es necesario, como lo indican Reyes-Jarquín y Hernández-Pozo (2012), hacer investigación empírica de cómo incrementar la autoeficacia en las personas. Se sugiere que en futuras investigaciones se utilice la hemoglobina glucosilada (HbA1c), que por limitaciones económicas no se pudo utilizar, ya que esto dará mayor validez a los resultados. 
La disparidad en los datos presentados de este estudio respecto a otros realizados en otras latitudes manifiesta la necesidad de considerar los aspectos socioculturales y las características del modelo de atención sanitaria como influyentes en el estudio de la adherencia terapéutica.

\section{NOTA DE RECONOCIMIENTO}

Este trabajo fue realizado gracias a la asistencia de las autoridades de la Unidad de Medicina Familiar No. 48 del Instituto Mexicano del Seguro Social, quienes otorgaron las facilidades para el acceso a la información necesaria para la realización de este estudio.

\section{REFERENCIAS BIBLIOGRÁFICAS}

Aljasem, L., Peyrot, M., Wissow, L. y Rubin, R. (2001).The impact of barriers and self-efficacy on self-care behaviors in type 2 diabetes. Diabetes Educator, 27(3), 393-404. doi: 10.1177/014572170102700309

Annesi, J., Unruh, J., Marti, C., Gorjala, S., y Tennant, G. (2011). Effects of the coach approach intervention on adherence to exercise in obese women: assessing mediation of social cognitive theory factors. Research Quarterly for Exercise and Sport, 82(1), 99-108. doi: 10.1080/02701367.2011.10599726

Arredondo, A., Márquez, E., Moreno, F., y Bazán, M. (2006). Influencia del apoyo social en el control del paciente diabético tipo 2. Revista de Especialidades Médico-Quirúrgicas, 11(3), 43-48.

Balcázar, P., Gurrola, G., Urcid, S., y Colín, H. (2011). Comparación de autoeficacia percibida en pacientes adultos con diabetes tipo 2 . Trabajo presentado en el Doceavo Congreso Virtual de Psiquiatría, Febrero-Marzo, Argentina.

Bandura, A. (2006). Toward of psychology of human agency. Perspectives on Psychology Science, 1(2), 164-180. doi: 10.1111/j.17456916.2006.00011.x

Bartfield, J., Ojehomon, N., Huskey, K., Davis, R. y Wee, C. (2010). Preferences and self-efficacy for diet modification among primary care patients. Obesity, 18(2), 430-432. doi: 10.1038/oby.2009.284

Dit, L., Baban, A. y Dumitrascu, D. (2012). Diabetes's adherence to treatment: the predictive value of satisfaction with medical care. Procedia - Social and Behavioral Sciences, 33, 508-512. doi:10.1016/j.sbspro.2012.01.173 
Escobedo, J. y Verdín, B. (1996). Incidencia y letalidad de las complicaciones agudas y crónicas de la diabetes mellitus. Salud Pública de México, 38(4), 236-242.

Ferrer, V., Gili, M., y Durán, A. (1999). La autoeficacia en la predicción de la adherencia al tratamiento de la diabetes. Revista de Psicología social aplicada, 9(2), 91-110.

Garay-Sevilla, M. E., Malacara, J. M., González-Contreras, E., WróbelZasada, K., Wróbel-Kaczmarczyk, K., y Gutiérrez-Roa, A. (2000). Perceived psychological stress in diabetes mellitus type 2. La Revista de Investigación Clínica, 52(3), 241-245.

Ginarte, Y. (2001). La adherencia terapéutica. Revista Cubana de Medicina General Integral, 17(5), 502-505.

Glasgow R. E., y Osteen V. L. (1992). Evaluating diabetes education: are we measuring the most important outcomes? Diabetes Care, 15, 1423-1432. doi: $10.2337 /$ diacare.15.10.1423

Harris, M. (2001). Racial and ethnic differences in health care acces and health outcomes for adults with type 2 Diabetes. Diabetes Care, 24(3), 454-459. doi: $10.2337 /$ diacare.24.3.454

Hurley A. C., y Shea C. A. (1992). Self-efficacy: strategy for enhancing diabetes self-care. The Diabetes Educator, 18(2), 146-150. doi:10.1177/014572179201800208

Jones, F., Harris, P., Waller, H. y Coggins, A. (2005). Adherence to an exercise prescription scheme: The role of expectations, self-efficacy, stage of change and psychological well-being. British Journal of Health Psychology, 10(3), 359-378. doi: 10.1348/135910704X24798

Lorig, K., Ritter, P. y Jacquez, A. (2005). Outcomes of border health Spanish/ English chronic disease self-management programs. The Diabetes Educator, 31(3), 401-409. doi: 10.1177/0145721705276574

Martín, L. (2004). Acerca del concepto de adherencia terapéutica. Revista Cubana Salud Pública, 32(3).

McCleary-Jones, V. (2011). Health literacy and its association with diabetes knowledge, self-efficacy and disease self-management among African Americans with diabetes mellitus. The ABNF Journal, 22(2), 25-32.

Nyunt, S., Howteerakul, N., Suwannapong, N. y Rajatanum, T. (2010). Selfefficacy, self-care beahviors and glycemic control among type-2 diabetes 
patients attending two private clinics in Yangon, Myanmar. Southeast Asian Journal of Tropical Medicine and Public Health, 41(4), 943-951.

O'Hea, E., Moon, S., Grothe, K., Boudreaux, E., Bodenlos, J., Wallston, K. y Brantley, P. (2009). The interaction of locus of control, self-efficacy, and outcome expectancy in relation to $\mathrm{HbAlc}$ in medically underserved individuals with type 2 diabetes. Journal of Behavioral Medicine, 32(1), 106-117. doi: 10.1007/s10865-008-9188-X

Organización Mundial de la Salud (2003). El incumplimiento del tratamiento prescrito para las enfermedades crónicas es un problema mundial de gran envergadura. Recuperado de http://www.who.int/mediacentre/ news/releases/2003/pr54/es/

Organización Mundial de la Salud (2011). Diabetes. Recuperado de http://www. who.int/mediacentre/factsheets/fs312/es/index.html

Plotnikoff, R., Brez, S., y Hotz, S. (2000). Exercise behavior in a community sample with diabetes: understanding the determinants of exercise behavioral change. The Diabetes Educator, 26(3), 450-459.

Quirós, D. y Villalobos, A. (2007). Comparación de factores vinculados a la adherencia al tratamiento de la diabetes mellitus tipo II entre una muestra urbana y otra rural de Costa Rica. Universitas Psychologica, 6(3), 679688.

Reyes-Jarquín, K. y Hernández-Pozo, M. (2012). Análisis crítico de los estudios que exploran la autoeficacia y bienestar vinculados al comportamiento saludable. Journal of Behavior, Health \& Social Issues, 3(2), 5-24.

Rose, M., Fliege, H., Hildebrandt, M., Schirop, T. y Klapp, B. (2002). The network of psychological variables in patients with diabetes and their importance for quality of life and metabolic control. Diabetes Care, 25(1), 35-42.

Sarkar, U., Fisher, L. y Schillinger, D. (2006). Is self-efficacy associated with diabetes self-management across race/ethnicity and health literacy? Diabetes Care, 29(4), 823-829.

Senecal, C., Nouwen, A. y White, D. (2000). Motivation and dietary self-care in adults with diabetes; are self-Efficacy and autonomous self-regulation complementary or competing constructs? Health Psychology, 19(5), 452457. doi: 10.10371/0278-6133.19.5.452

Stanford Patient Education Research Center (2004). Spanish Diabetes SelfEfficacy. Recuperado de http://patienteducation.stanford.edu/research/ sediabetesesp.html 
Vázquez, J., y Panduro, A. (2001). Diabetes Mellitus tipo 2: un problema epidemiológico y de emergencia en México. Investigación en Salud, 3(99), 18-26.

Villalobos, A. y Araya, C. (2001). Variables que afectan la adherencia al tratamiento en la diabetes mellitus tipo II y características poblacionales de una muestra de la zona norte de Costa Rica. Revista Costarricense de Psicología, 33, 19-37.

Villalobos, A., Brenes, J., Quirós, D. y, León, G. (2006). Características psicométricas de la Escala de Adherencia al Tratamiento de la Diabetes Mellitus Tipo II-Versión III (EATDM - III) en una muestra de pacientes diabéticos de Costa Rica. Acta Colombiana de Psicología, 9(2), 31-38.

Villalpando, S., De la Cruz, V., Rojas, R., Shamah-Levy, T., Ávila, M., Gaona, B. y cols. (2010). Prevalence and distribution of type 2 diabetes mellitus in Mexican adult population. A probabilistic survey. Salud Pública de México, 52(1), 19-26.

Warziski, M., Sereika, S., Styn, M., Music, E. y Burke, L. (2008). Changes in self-efficacy and dietary adherence: the impact on weight loss in the PREFER study. Journal of Behavioral Medicine, 31(1), 81-92. doi: $10.1007 / \mathrm{s} 10865-007-9135-2$

Wu, S-FV., Courtney, M., Edwards, H., McDowell, J., Shortridge-Baggett, LM., y Chang, P-J. (2007). Self-efficacy outcome expectations and selfcare behavior in people with type 2 diabetes in Taiwan. Journal of Nursing and Healthcare of Chronic Illness in association with Journal of Clinical Nursing, 16(11), 250-257. doi: 10.1111/j.1365-2702.2006.01930.x 